\title{
Simultaneous Search and Efficiency of Entry and Search Intensity
}

\author{
By Pieter A. Gautier and Christian L. Holzner*
}

This web appendix contains some of the proofs of the paper:

Gautier, P. and C. Holzner (2016), Simultaneous search and efficiency of entry and search intensity, American Economic Journal: Microeconomics, forthcoming.

\section{Web Appendix}

\section{A. Full Proof of Proposition 1}

The social planner maximizes

$$
\begin{gathered}
\max _{C, p_{c}, v_{c}}\left[u-u \frac{z_{c}\left(t^{*}\right)}{z_{c}(0)}\left(1-e^{-z_{c}\left(t^{*}\right)}\right)+\sum_{c \in C} v_{c}\left(1-e^{-z_{c}\left(t^{*}\right)}-z_{c}\left(t^{*}\right) e^{-z_{c}\left(t^{*}\right)}\right)\right] \\
\text { s.t. } \prod_{i \in C}\left(\frac{z_{i}\left(t^{*}\right)}{z_{i}(0)}\right)^{\frac{a}{a-1} p_{i}}=\frac{z_{c}\left(t^{*}\right)}{z_{c}(0)}\left(1-e^{-z_{c}\left(t^{*}\right)}\right),
\end{gathered}
$$

and $\sum_{c \in C} v_{c}=v$ and $\sum_{c \in C} p_{c}=1$.

We start by showing that random search is optimal in case of two submarket of vacancies $r$ and $b$. The general result then follows by induction. Denote $M=t^{*}+\min \left[u\left(t^{*}\right), \sum_{c \in\{r, b\}} v_{c}\left(t^{*}\right)\right]$. Using the results of Lemma 4 we get

$$
\begin{aligned}
M & =u-u\left(\left(\frac{z_{r}\left(t^{*}\right)}{z_{r}(0)}\right)^{p_{r}}\left(\frac{z_{b}\left(t^{*}\right)}{z_{b}(0)}\right)^{p_{b}}\right)^{\frac{a}{a-1}} \\
& +\sum_{c \in\{r, b\}} v_{c}\left(1-e^{-z_{c}\left(t^{*}\right)}-z_{c}\left(t^{*}\right) e^{-z_{c}\left(t^{*}\right)}\right),
\end{aligned}
$$

where $v_{r}=v-v_{b}$ and $p_{r}=1-p_{b}$.

* Gautier: VU University Amsterdam, Tinbergen Institute email: p.a.gautier@vu.nl. Holzner: University of Munich, Ifo Institute for Economic research and CESifo, address2, holzner@ifo.de. Acknowledgements: The authors gratefully acknowledge the hospitality of CESifo and Christian Holzner acknowledges the financial support by the German Research Foundation, grant Ho 4537/1-1. The authors thank Xiaoming Cai, Philipp Kircher, Mihai Manea, Ren van den Brink and seminar participants at Cambridge, CESifo, CREST, the University of Edinburgh, the University of Essex, the University of Konstanz, the University of Mainz, NBER, the Norwegian School of Management, University of Helsinki the 2011 Tinbergen conference, the University of Amsterdam and the Vrije Universiteit Amsterdam 
The FOC's with respect to $v_{b}$ is given by,

(1)

$$
\begin{aligned}
\frac{\partial M}{\partial v_{b}} & =0 \\
& =-u \frac{a}{a-1} \Psi\left(\frac{p_{b}}{v_{b}}-\frac{p_{r}}{v_{r}}\right) \\
& -u \frac{a}{a-1} \Psi\left(\frac{p_{b}}{z_{b}\left(t^{*}\right)}\left(\frac{d z_{b}}{d v_{b}}-\frac{d z_{b}}{d v_{r}}\right)-\frac{p_{r}}{z_{r}\left(t^{*}\right)}\left(\frac{d z_{r}}{d v_{r}}-\frac{d z_{r}}{d v_{b}}\right)\right) \\
& +\left(1-e^{-z_{b}\left(t^{*}\right)}-z_{b}\left(t^{*}\right) e^{-z_{b}\left(t^{*}\right)}\right)-\left(1-e^{-z_{r}\left(t^{*}\right)}-z_{r}\left(t^{*}\right) e^{-z_{r}\left(t^{*}\right)}\right) \\
& +v_{b} z_{b}\left(t^{*}\right) e^{-z_{b}\left(t^{*}\right)}\left(\frac{d z_{b}}{d v_{b}}-\frac{d z_{b}}{d v_{r}}\right)-v_{r} z_{r}\left(t^{*}\right) e^{-z_{r}\left(t^{*}\right)}\left(\frac{d z_{r}}{d v_{r}}-\frac{d z_{r}}{d v_{b}}\right) \\
& =-u \frac{a}{a-1} \Psi\left(\frac{p_{b}}{v_{b}}-\frac{p_{r}}{v_{r}}\right) \\
& +z_{b}(0) v_{b}\left(\frac{z_{b}\left(t^{*}\right) e^{-z_{b}\left(t^{*}\right)}}{z_{b}(0)}-\frac{1}{a-1} \frac{1-e^{-z_{b}\left(t^{*}\right)}}{z_{b}(0)}\right)\left(\frac{d z_{b}}{d v_{b}}-\frac{d z_{b}}{d v_{r}}\right) \\
& -z_{r}(0) v_{r}\left(\frac{z_{r}\left(t^{*}\right) e^{-z_{r}\left(t^{*}\right)}}{z_{r}(0)}-\frac{1}{a-1} \frac{1-e^{-z_{r}\left(t^{*}\right)}}{z_{r}(0)}\right)\left(\frac{d z_{r}}{d v_{r}}-\frac{d z_{r}}{d v_{b}}\right) \\
& +\left(1-e^{-z_{b}\left(t^{*}\right)}-z_{b}\left(t^{*}\right) e^{-z_{b}\left(t^{*}\right)}\right)-\left(1-e^{-z_{r}\left(t^{*}\right)}-z_{r}\left(t^{*}\right) e^{-z_{r}\left(t^{*}\right)}\right),
\end{aligned}
$$

with

$$
\begin{aligned}
\Psi & =\left(\left(\frac{z_{r}\left(t^{*}\right)}{z_{r}(0)}\right)^{p_{r}}\left(\frac{z_{b}\left(t^{*}\right)}{z_{b}(0)}\right)^{p_{b}}\right)^{\frac{a}{a-1}} \text { and }, \\
\frac{d z_{c}}{d v_{k}} & =\frac{d z_{c}\left(t^{*}\right)}{d v_{k}} \text { with } c, k \in\{r, b\} .
\end{aligned}
$$

According to equation (32) in the main text the following two functions determine $z_{r}\left(t^{*}\right)$ and $z_{b}\left(t^{*}\right)$ simultaneously, i.e.,

$$
\begin{aligned}
& R=\left(\left(\frac{z_{r}\left(t^{*}\right)}{z_{r}(0)}\right)^{p_{r}}\left(\frac{z_{b}\left(t^{*}\right)}{z_{b}(0)}\right)^{p_{b}}\right)^{\frac{a}{a-1}}-\frac{z_{r}\left(t^{*}\right)\left(1-e^{-z_{r}\left(t^{*}\right)}\right)}{z_{r}(0)}=0, \\
& B=\left(\left(\frac{z_{r}\left(t^{*}\right)}{z_{r}(0)}\right)^{p_{r}}\left(\frac{z_{b}\left(t^{*}\right)}{z_{b}(0)}\right)^{p_{b}}\right)^{\frac{a}{a-1}}-\frac{z_{b}\left(t^{*}\right)\left(1-e^{-z_{b}\left(t^{*}\right)}\right)}{z_{b}(0)}=0 .
\end{aligned}
$$

Using the implicit function theorem allows us to determine the derivatives of $z_{r}\left(t^{*}\right)$ and $z_{b}\left(t^{*}\right)$ with respect to $v_{b}, v_{r}$ and $p_{b}, p_{r}$, i.e., 


$$
\begin{aligned}
\frac{d z_{r}}{d v_{b}} & =\frac{B_{v_{b}}^{\prime} R_{z_{b}}^{\prime}-B_{z_{b}}^{\prime} R_{v_{b}}^{\prime}}{B_{z_{b}}^{\prime} R_{z_{r}}^{\prime}-B_{z_{r}}^{\prime} R_{z_{b}}^{\prime}}, \text { and } \frac{d z_{b}}{d v_{r}}=\frac{B_{z_{r}}^{\prime} R_{v_{r}}^{\prime}-B_{v_{r}}^{\prime} R_{z_{r}}^{\prime}}{B_{z_{b}}^{\prime} R_{z_{r}}^{\prime}-B_{z_{r}}^{\prime} R_{z_{b}}^{\prime}}, \\
\frac{d z_{b}}{d v_{b}} & =\frac{B_{z_{r}}^{\prime} R_{v_{b}}^{\prime}-B_{v_{b}}^{\prime} R_{z_{r}}^{\prime}}{B_{z_{b}}^{\prime} R_{z_{r}}^{\prime}-B_{z_{r}}^{\prime} R_{z_{b}}^{\prime}}, \text { and } \frac{d z_{r}}{d v_{r}}=\frac{B_{v_{r}}^{\prime} R_{z_{b}}^{\prime}-B_{z_{b}}^{\prime} R_{v_{r}}^{\prime}}{B_{z_{b}}^{\prime} R_{z_{r}}^{\prime}-B_{z_{r}}^{\prime} R_{z_{b}}^{\prime}},
\end{aligned}
$$

Using equations (4) to (5) we get,

$$
\begin{aligned}
& \frac{d z_{b}}{d v_{b}}-\frac{d z_{b}}{d v_{r}}=\frac{B_{z_{r}}^{\prime} R_{v_{b}}^{\prime}-B_{v_{b}}^{\prime} R_{z_{r}}^{\prime}-B_{z_{r}}^{\prime} R_{v_{r}}^{\prime}+B_{v_{r}}^{\prime} R_{z_{r}}^{\prime}}{B_{z_{b}}^{\prime} R_{z_{r}}^{\prime}-B_{z_{r}}^{\prime} R_{z_{b}}^{\prime}} \\
& \frac{d z_{r}}{d v_{r}}-\frac{d z_{r}}{d v_{b}}=\frac{B_{v_{r}}^{\prime} R_{z_{b}}^{\prime}-B_{z_{b}}^{\prime} R_{v_{r}}^{\prime}-B_{v_{b}}^{\prime} R_{z_{b}}^{\prime}+B_{z_{b}}^{\prime} R_{v_{b}}^{\prime}}{B_{z_{b}}^{\prime} R_{z_{r}}^{\prime}-B_{z_{r}}^{\prime} R_{z_{b}}^{\prime}}
\end{aligned}
$$

Using equations (2) and (3) we can obtain the following expressions for the derivatives of $R$ and $B$, i.e.,

$$
\begin{array}{ll}
R_{v_{b}}^{\prime}=p_{b} \frac{a}{a-1} \frac{1}{v_{b}} \Psi, & R_{v_{r}}^{\prime}=p_{r} \frac{a}{a-1} \frac{1}{v_{r}} \Psi-\frac{1}{v_{r}} \frac{z_{r}\left(t^{*}\right)\left(1-e^{-z_{r}\left(t^{*}\right)}\right)}{z_{r}(0)}, \\
B_{v_{r}}^{\prime}=p_{r} \frac{a}{a-1} \frac{1}{v_{r}} \Psi, & B_{v_{b}}^{\prime}=p_{b} \frac{a}{a-1} \frac{1}{v_{b}} \Psi-\frac{1}{v_{b}} \frac{z_{b}\left(t^{*}\right)\left(1-e^{-z_{b}\left(t^{*}\right)}\right)}{z_{b}(0)}, \\
R_{z_{b}}^{\prime}=p_{b} \frac{a}{a-1} \frac{1}{z_{b}\left(t^{*}\right)} \Psi, & R_{z_{r}}^{\prime}=p_{r} \frac{a}{a-1} \frac{1}{z_{r}\left(t^{*}\right)} \Psi-\frac{1-e^{-z_{r}\left(t^{*}\right)}+z_{r}\left(t^{*}\right) e^{-z_{r}\left(t^{*}\right)}}{z_{r}(0)}, \\
B_{z_{r}}^{\prime}=p_{r} \frac{a}{a-1} \frac{1}{z_{r}\left(t^{*}\right)} \Psi, & B_{z_{b}}^{\prime}=p_{b} \frac{a}{a-1} \frac{1}{z_{b}\left(t^{*}\right)} \Psi-\frac{1-e^{-z_{b}\left(t^{*}\right)}+z_{b}\left(t^{*}\right) e^{-z_{b}\left(t^{*}\right)}}{z_{b}(0)} .
\end{array}
$$

We therefore get the following expressions for,

$$
\begin{aligned}
& B_{z_{r}}^{\prime} R_{v_{b}}^{\prime}-B_{v_{b}}^{\prime} R_{z_{r}}^{\prime}-B_{z_{r}}^{\prime} R_{v_{r}}^{\prime}+B_{v_{r}}^{\prime} R_{z_{r}}^{\prime} \\
& =p_{r} \frac{a}{a-1} \frac{1}{z_{r}\left(t^{*}\right)} \Psi\left(\frac{1}{v_{r}} \frac{z_{r}\left(t^{*}\right)\left(1-e^{-z_{r}\left(t^{*}\right)}\right)}{z_{r}(0)}+\frac{1}{v_{b}} \frac{z_{b}\left(t^{*}\right)\left(1-e^{-z_{b}\left(t^{*}\right)}\right)}{z_{b}(0)}\right) \\
& +\left(\frac{p_{b}}{v_{b}}-\frac{p_{r}}{v_{r}}\right) \frac{a}{a-1} \Psi \frac{1-e^{-z_{r}\left(t^{*}\right)}+z_{r}\left(t^{*}\right) e^{-z_{r}\left(t^{*}\right)}}{z_{r}(0)} \\
& -\frac{1}{v_{b}} \frac{z_{b}\left(t^{*}\right)\left(1-e^{-z_{b}\left(t^{*}\right)}\right)}{z_{b}(0)} \frac{1-e^{-z_{r}\left(t^{*}\right)}+z_{r}\left(t^{*}\right) e^{-z_{r}\left(t^{*}\right)}}{z_{r}(0)},
\end{aligned}
$$




$$
\begin{aligned}
& B_{v_{r}}^{\prime} R_{z_{b}}^{\prime}-B_{z_{b}}^{\prime} R_{v_{r}}^{\prime}-B_{v_{b}}^{\prime} R_{z_{b}}^{\prime}+B_{z_{b}}^{\prime} R_{v_{b}}^{\prime} \\
& =p_{b} \frac{a}{a-1} \frac{1}{z_{b}\left(t^{*}\right)} \Psi\left(\frac{1}{v_{r}} \frac{z_{r}\left(t^{*}\right)\left(1-e^{-z_{r}\left(t^{*}\right)}\right)}{z_{r}(0)}+\frac{1}{v_{b}} \frac{z_{b}\left(t^{*}\right)\left(1-e^{-z_{b}\left(t^{*}\right)}\right)}{z_{b}(0)}\right) \\
& +\left(\frac{p_{r}}{v_{r}}-\frac{p_{b}}{v_{b}}\right) \frac{a}{a-1} \Psi \frac{1-e^{-z_{b}\left(t^{*}\right)}+z_{b}\left(t^{*}\right) e^{-z_{b}\left(t^{*}\right)}}{z_{b}(0)} \\
& -\frac{1}{v_{r}} \frac{z_{r}\left(t^{*}\right)\left(1-e^{-z_{r}\left(t^{*}\right)}\right)}{z_{r}(0)} \frac{1-e^{-z_{b}\left(t^{*}\right)}+z_{b}\left(t^{*}\right) e^{-z_{b}\left(t^{*}\right)}}{z_{b}(0)}, \\
& B_{z_{b}}^{\prime} R_{z_{r}}^{\prime}-B_{z_{r}}^{\prime} R_{z_{b}}^{\prime} \\
& =p_{r} \frac{1-e^{-z_{b}\left(t^{*}\right)}+z_{b}\left(t^{*}\right) e^{-z_{b}\left(t^{*}\right)}}{z_{b}(0)}\left(\frac{z_{r}\left(t^{*}\right) e^{-z_{r}\left(t^{*}\right)}}{z_{r}(0)}-\frac{1}{a-1} \frac{1-e^{-z_{r}\left(t^{*}\right)}}{z_{r}(0)}\right) \\
& +p_{b} \frac{1-e^{-z_{r}\left(t^{*}\right)}+z_{r}\left(t^{*}\right) e^{-z_{r}\left(t^{*}\right)}}{z_{r}(0)}\left(\frac{z_{b}\left(t^{*}\right) e^{-z_{b}\left(t^{*}\right)}}{z_{b}(0)}-\frac{1}{a-1} \frac{1-e^{-z_{b}\left(t^{*}\right)}}{z_{b}(0)}\right)
\end{aligned}
$$

Multiplying the FOC with $\left(B_{z_{b}}^{\prime} R_{z_{r}}^{\prime}-B_{z_{r}}^{\prime} R_{z_{b}}^{\prime}\right)$ and substituting $\left(\frac{d z_{b}}{d v_{b}}-\frac{d z_{b}}{d v_{r}}\right)$ and $\left(\frac{d z_{r}}{d v_{r}}-\frac{d z_{r}}{d v_{b}}\right)$ using the equalities (2) and (3) gives,

$$
\begin{aligned}
& 0=-u \frac{a}{a-1} \Psi\left(\frac{p_{b}}{v_{b}}-\frac{p_{r}}{v_{r}}\right) p_{r} \frac{1-e^{-z_{b}\left(t^{*}\right)}+z_{b}\left(t^{*}\right) e^{-z_{b}\left(t^{*}\right)}}{z_{b}(0)}\left(\frac{z_{r}\left(t^{*}\right) e^{-z_{r}\left(t^{*}\right)}}{z_{r}(0)}-\frac{1}{a-1} \frac{1-e^{-z_{r}\left(t^{*}\right)}}{z_{r}(0)}\right) \\
& -u \frac{a}{a-1} \Psi\left(\frac{p_{b}}{v_{b}}-\frac{p_{r}}{v_{r}}\right) p_{b} \frac{1-e^{-z_{r}\left(t^{*}\right)}+z_{r}\left(t^{*}\right) e^{-z_{r}\left(t^{*}\right)}}{z_{r}(0)}\left(\frac{z_{b}\left(t^{*}\right) e^{-z_{b}\left(t^{*}\right)}}{z_{b}(0)}-\frac{1}{a-1} \frac{1-e^{-z_{b}\left(t^{*}\right)}}{z_{b}(0)}\right) \\
& +z_{b}(0) v_{b}\left(\frac{z_{b}\left(t^{*}\right) e^{-z_{b}\left(t^{*}\right)}}{z_{b}(0)}-\frac{1}{a-1} \frac{1-e^{-z_{b}\left(t^{*}\right)}}{z_{b}(0)}\right) p_{r} \frac{a}{a-1} \frac{\left(1-e^{-z_{r}\left(t^{*}\right)}\right)}{z_{r}(0)} \Psi\left(\frac{1}{v_{r}}+\frac{1}{v_{b}}\right) \\
& +z_{b}(0) v_{b}\left(\frac{z_{b}\left(t^{*}\right) e^{-z_{b}\left(t^{*}\right)}}{z_{b}(0)}-\frac{1}{a-1} \frac{1-e^{-z_{b}\left(t^{*}\right)}}{z_{b}(0)}\right)\left(\frac{p_{b}}{v_{b}}-\frac{p_{r}}{v_{r}}\right) \frac{a}{a-1} \Psi \frac{1-e^{-z_{r}\left(t^{*}\right)}+z_{r}\left(t^{*}\right) e^{-z_{r}\left(t^{*}\right)}}{z_{r}(0)} \\
& -z_{b}(0) v_{b}\left(\frac{z_{b}\left(t^{*}\right) e^{-z_{b}\left(t^{*}\right)}}{z_{b}(0)}-\frac{1}{a-1} \frac{1-e^{-z_{b}\left(t^{*}\right)}}{z_{b}(0)}\right) \frac{1}{v_{b}} \frac{z_{b}\left(t^{*}\right)\left(1-e^{-z_{b}\left(t^{*}\right)}\right)}{z_{b}(0)} \frac{1-e^{-z_{r}\left(t^{*}\right)}+z_{r}\left(t^{*}\right) e^{-z_{r}\left(t^{*}\right)}}{z_{r}(0)} \\
& -z_{r}(0) v_{r}\left(\frac{z_{r}\left(t^{*}\right) e^{-z_{r}\left(t^{*}\right)}}{z_{r}(0)}-\frac{1}{a-1} \frac{1-e^{-z_{r}\left(t^{*}\right)}}{z_{r}(0)}\right) p_{b} \frac{a}{a-1} \frac{\left(1-e^{-z_{b}\left(t^{*}\right)}\right)}{z_{b}(0)} \Psi\left(\frac{1}{v_{r}}+\frac{1}{v_{b}}\right) \\
& -z_{r}(0) v_{r}\left(\frac{z_{r}\left(t^{*}\right) e^{-z_{r}\left(t^{*}\right)}}{z_{r}(0)}-\frac{1}{a-1} \frac{1-e^{-z_{r}\left(t^{*}\right)}}{z_{r}(0)}\right)\left(\frac{p_{r}}{v_{r}}-\frac{p_{b}}{v_{b}}\right) \frac{a}{a-1} \Psi \frac{1-e^{-z_{b}\left(t^{*}\right)}+z_{b}\left(t^{*}\right) e^{-z_{b}\left(t^{*}\right)}}{z_{b}(0)} \\
& +z_{r}(0) v_{r}\left(\frac{z_{r}\left(t^{*}\right) e^{-z_{r}\left(t^{*}\right)}}{z_{r}(0)}-\frac{1}{a-1} \frac{1-e^{-z_{r}\left(t^{*}\right)}}{z_{r}(0)}\right) \frac{1}{v_{r}} \frac{z_{r}\left(t^{*}\right)\left(1-e^{-z_{r}\left(t^{*}\right)}\right)}{z_{r}(0)} \frac{1-e^{-z_{b}\left(t^{*}\right)}+z_{b}\left(t^{*}\right) e^{-z_{b}\left(t^{*}\right)}}{z_{b}(0)} \\
& +\left[\left(1-e^{-z_{b}\left(t^{*}\right)}-z_{b}\left(t^{*}\right) e^{-z_{b}\left(t^{*}\right)}\right)-\left(1-e^{-z_{r}\left(t^{*}\right)}-z_{r}\left(t^{*}\right) e^{-z_{r}\left(t^{*}\right)}\right)\right]\left(B_{z_{b}}^{\prime} R_{z_{r}}^{\prime}-B_{z_{r}}^{\prime} R_{z_{b}}^{\prime}\right),
\end{aligned}
$$


Rearranging implies,

$$
\begin{aligned}
0 & =-p_{r} a u\left(\frac{z_{r}\left(t^{*}\right) e^{-z_{r}\left(t^{*}\right)}}{z_{r}(0)}-\frac{1}{a-1} \frac{1-e^{-z_{r}\left(t^{*}\right)}}{z_{r}(0)}\right) \frac{1}{a-1} \Psi\left(\frac{p_{b}}{v_{b}}-\frac{p_{r}}{v_{r}}\right) \frac{1-e^{-z_{b}\left(t^{*}\right)}+z_{b}\left(t^{*}\right) e^{-z_{b}\left(t^{*}\right)}}{z_{b}(0)} \\
& -p_{r} a u\left(\frac{z_{r}\left(t^{*}\right) e^{-z_{r}\left(t^{*}\right)}}{z_{r}(0)}-\frac{1}{a-1} \frac{1-e^{-z_{r}\left(t^{*}\right)}}{z_{r}(0)}\right) p_{b} \frac{a}{a-1} \frac{\left(1-e^{-z_{b}\left(t^{*}\right)}\right)}{z_{b}(0)} \Psi\left(\frac{1}{v_{r}}+\frac{1}{v_{b}}\right) \\
& -p_{r} a u\left(\frac{z_{r}\left(t^{*}\right) e^{-z_{r}\left(t^{*}\right)}}{z_{r}(0)}-\frac{1}{a-1} \frac{1-e^{-z_{r}\left(t^{*}\right)}}{z_{r}(0)}\right)\left(\frac{p_{r}}{v_{r}}-\frac{p_{b}}{v_{b}}\right) \frac{a}{a-1} \Psi \frac{1-e^{-z_{b}\left(t^{*}\right)}+z_{b}\left(t^{*}\right) e^{-z_{b}\left(t^{*}\right)}}{z_{b}(0)} \\
& +p_{r} a u\left(\frac{z_{r}\left(t^{*}\right) e^{-z_{r}\left(t^{*}\right)}}{z_{r}(0)}-\frac{1}{a-1} \frac{1-e^{-z_{r}\left(t^{*}\right)}}{z_{r}(0)}\right) \frac{1}{v_{r}} \Psi \frac{1-e^{-z_{b}\left(t^{*}\right)}+z_{b}\left(t^{*}\right) e^{-z_{b}\left(t^{*}\right)}}{z_{b}(0)} \\
& -p_{b} u a\left(\frac{z_{b}\left(t^{*}\right) e^{-z_{b}\left(t^{*}\right)}}{z_{b}(0)}-\frac{1}{a-1} \frac{1-e^{-z_{b}\left(t^{*}\right)}}{z_{b}(0)}\right) \frac{1}{a-1} \Psi\left(\frac{p_{b}}{v_{b}}-\frac{p_{r}}{v_{r}}\right) \frac{1-e^{-z_{r}\left(t^{*}\right)}+z_{r}\left(t^{*}\right) e^{-z_{r}\left(t^{*}\right)}}{z_{r}(0)} \\
& +p_{b} a u\left(\frac{z_{b}\left(t^{*}\right) e^{-z_{b}\left(t^{*}\right)}}{z_{b}(0)}-\frac{1}{a-1} \frac{1-e^{-z_{b}\left(t^{*}\right)}}{z_{b}(0)}\right) p_{r} \frac{a}{a-1} \frac{\left(1-e^{-z_{r}\left(t^{*}\right)}\right)}{z_{r}(0)} \Psi\left(\frac{1}{v_{r}}+\frac{1}{v_{b}}\right) \\
& +p_{b} a u\left(\frac{z_{b}\left(t^{*}\right) e^{-z_{b}\left(t^{*}\right)}}{z_{b}(0)}-\frac{1}{a-1} \frac{1-e^{-z_{b}\left(t^{*}\right)}}{z_{b}(0)}\right)\left(\frac{p_{b}}{v_{b}}-\frac{p_{r}}{v_{r}}\right) \frac{a}{a-1} \Psi \frac{1-e^{-z_{r}\left(t^{*}\right)}+z_{r}\left(t^{*}\right) e^{-z_{r}\left(t^{*}\right)}}{z_{r}(0)} \\
& -p_{b} a u\left(\frac{z_{b}\left(t^{*}\right) e^{-z_{b}\left(t^{*}\right)}}{z_{b}(0)}-\frac{1}{a-1} \frac{1-e^{-z_{b}\left(t^{*}\right)}}{z_{b}(0)}\right) \frac{1}{v_{b}} \Psi \frac{1-e^{-z_{r}\left(t^{*}\right)}+z_{r}\left(t^{*}\right) e^{-z_{r}\left(t^{*}\right)}}{z_{r}(0)} \\
& +\left[\left(1-e^{-z_{b}\left(t^{*}\right)}-z_{b}\left(t^{*}\right) e^{-z_{b}\left(t^{*}\right)}\right)-\left(1-e^{-z_{r}\left(t^{*}\right)}-z_{r}\left(t^{*}\right) e^{-z_{r}\left(t^{*}\right)}\right)\right]\left(B_{z_{b}}^{\prime} R_{z_{r}}^{\prime}-B_{z_{r}}^{\prime} R_{z_{b}}^{\prime}\right) .
\end{aligned}
$$

Simplifying implies,

$$
\begin{aligned}
0 & =p_{r} a u\left(\frac{z_{r}\left(t^{*}\right) e^{-z_{r}\left(t^{*}\right)}}{z_{r}(0)}-\frac{1}{a-1} \frac{1-e^{-z_{r}\left(t^{*}\right)}}{z_{r}(0)}\right)\left(\frac{1}{v_{r}}+\frac{p_{b}}{v_{b}}-\frac{p_{r}}{v_{r}}\right) \Psi \frac{1-e^{-z_{b}\left(t^{*}\right)}+z_{b}\left(t^{*}\right) e^{-z_{b}\left(t^{*}\right)}}{z_{b}(0)} \\
& -p_{r} a u\left(\frac{z_{r}\left(t^{*}\right) e^{-z_{r}\left(t^{*}\right)}}{z_{r}(0)}-\frac{1}{a-1} \frac{1-e^{-z_{r}\left(t^{*}\right)}}{z_{r}(0)}\right) p_{b} \frac{a}{a-1} \frac{\left(1-e^{-z_{b}\left(t^{*}\right)}\right)}{z_{b}(0)} \Psi\left(\frac{1}{v_{r}}+\frac{1}{v_{b}}\right) \\
& +p_{b} u a\left(\frac{z_{b}\left(t^{*}\right) e^{-z_{b}\left(t^{*}\right)}}{z_{b}(0)}-\frac{1}{a-1} \frac{1-e^{-z_{b}\left(t^{*}\right)}}{z_{b}(0)}\right)\left(-\frac{1}{v_{b}}+\frac{p_{b}}{v_{b}}-\frac{p_{r}}{v_{r}}\right) \Psi \frac{1-e^{-z_{r}\left(t^{*}\right)}+z_{r}\left(t^{*}\right) e^{-z_{r}\left(t^{*}\right)}}{z_{r}(0)} \\
& +p_{b} a u\left(\frac{z_{b}\left(t^{*}\right) e^{-z_{b}\left(t^{*}\right)}}{z_{b}(0)}-\frac{1}{a-1} \frac{1-e^{-z_{b}\left(t^{*}\right)}}{z_{b}(0)}\right) p_{r} \frac{a}{a-1} \frac{\left(1-e^{-z_{r}\left(t^{*}\right)}\right)}{z_{r}(0)} \Psi\left(\frac{1}{v_{r}}+\frac{1}{v_{b}}\right) \\
& +\left[\left(1-e^{-z_{b}\left(t^{*}\right)}-z_{b}\left(t^{*}\right) e^{-z_{b}\left(t^{*}\right)}\right)-\left(1-e^{-z_{r}\left(t^{*}\right)}-z_{r}\left(t^{*}\right) e^{-z_{r}\left(t^{*}\right)}\right)\right]\left(B_{z_{b}}^{\prime} R_{z_{r}}^{\prime}-B_{z_{r}}^{\prime} R_{z_{b}}^{\prime}\right) .
\end{aligned}
$$


Using the fact that $1=p_{b}+p_{r}$ gives,

$$
\begin{aligned}
0 & =p_{r} a u\left(\frac{z_{r}\left(t^{*}\right) e^{-z_{r}\left(t^{*}\right)}}{z_{r}(0)}-\frac{1}{a-1} \frac{1-e^{-z_{r}\left(t^{*}\right)}}{z_{r}(0)}\right) p_{b}\left(\frac{1}{v_{r}}+\frac{1}{v_{b}}\right) \Psi \frac{1-e^{-z_{b}\left(t^{*}\right)}+z_{b}\left(t^{*}\right) e^{-z_{b}\left(t^{*}\right)}}{z_{b}(0)} \\
& -p_{r} a u\left(\frac{z_{r}\left(t^{*}\right) e^{-z_{r}\left(t^{*}\right)}}{z_{r}(0)}-\frac{1}{a-1} \frac{1-e^{-z_{r}\left(t^{*}\right)}}{z_{r}(0)}\right) p_{b} \frac{a}{a-1} \frac{\left(1-e^{-z_{b}\left(t^{*}\right)}\right)}{z_{b}(0)} \Psi\left(\frac{1}{v_{r}}+\frac{1}{v_{b}}\right) \\
& -p_{b} u a\left(\frac{z_{b}\left(t^{*}\right) e^{-z_{b}\left(t^{*}\right)}}{z_{b}(0)}-\frac{1}{a-1} \frac{1-e^{-z_{b}\left(t^{*}\right)}}{z_{b}(0)}\right) p_{r}\left(\frac{1}{v_{b}}+\frac{1}{v_{r}}\right) \Psi \frac{1-e^{-z_{r}\left(t^{*}\right)}+z_{r}\left(t^{*}\right) e^{-z_{r}\left(t^{*}\right)}}{z_{r}(0)} \\
& +p_{b} a u\left(\frac{z_{b}\left(t^{*}\right) e^{-z_{b}\left(t^{*}\right)}}{z_{b}(0)}-\frac{1}{a-1} \frac{1-e^{-z_{b}\left(t^{*}\right)}}{z_{b}(0)}\right) p_{r} \frac{a}{a-1} \frac{\left(1-e^{-z_{r}\left(t^{*}\right)}\right)}{z_{r}(0)} \Psi\left(\frac{1}{v_{r}}+\frac{1}{v_{b}}\right) \\
& +\left[\left(1-e^{-z_{b}\left(t^{*}\right)}-z_{b}\left(t^{*}\right) e^{-z_{b}\left(t^{*}\right)}\right)-\left(1-e^{-z_{r}\left(t^{*}\right)}-z_{r}\left(t^{*}\right) e^{-z_{r}\left(t^{*}\right)}\right)\right]\left(B_{z_{b}}^{\prime} R_{z_{r}}^{\prime}-B_{z_{r}}^{\prime} R_{z_{b}}^{\prime}\right) .
\end{aligned}
$$

Simplifying gives,

$$
\begin{aligned}
0 & =p_{r} a u\left(\frac{z_{r}\left(t^{*}\right) e^{-z_{r}\left(t^{*}\right)}}{z_{r}(0)}-\frac{1}{a-1} \frac{1-e^{-z_{r}\left(t^{*}\right)}}{z_{r}(0)}\right) p_{b}\left(\frac{1}{v_{r}}+\frac{1}{v_{b}}\right) \Psi\left(\frac{z_{b}\left(t^{*}\right) e^{-z_{b}\left(t^{*}\right)}}{z_{b}(0)}-\frac{1}{a-1} \frac{1-e^{-z_{b}\left(t^{*}\right)}}{z_{b}(0)}\right) \\
& -p_{b} u a\left(\frac{z_{b}\left(t^{*}\right) e^{-z_{b}\left(t^{*}\right)}}{z_{b}(0)}-\frac{1}{a-1} \frac{1-e^{-z_{b}\left(t^{*}\right)}}{z_{b}(0)}\right) p_{r}\left(\frac{1}{v_{b}}+\frac{1}{v_{r}}\right) \Psi\left(\frac{z_{r}\left(t^{*}\right) e^{-z_{r}\left(t^{*}\right)}}{z_{r}(0)}-\frac{1}{a-1} \frac{1-e^{-z_{r}\left(t^{*}\right)}}{z_{r}(0)}\right) \\
& +\left[\left(1-e^{-z_{b}\left(t^{*}\right)}-z_{b}\left(t^{*}\right) e^{-z_{b}\left(t^{*}\right)}\right)-\left(1-e^{-z_{r}\left(t^{*}\right)}-z_{r}\left(t^{*}\right) e^{-z_{r}\left(t^{*}\right)}\right)\right]\left(B_{z_{b}}^{\prime} R_{z_{r}}^{\prime}-B_{z_{r}}^{\prime} R_{z_{b}}^{\prime}\right), \\
& =\left[\left(1-e^{-z_{b}\left(t^{*}\right)}-z_{b}\left(t^{*}\right) e^{-z_{b}\left(t^{*}\right)}\right)-\left(1-e^{-z_{r}\left(t^{*}\right)}-z_{r}\left(t^{*}\right) e^{-z_{r}\left(t^{*}\right)}\right)\right]\left(B_{z_{b}}^{\prime} R_{z_{r}}^{\prime}-B_{z_{r}}^{\prime} R_{z_{b}}^{\prime}\right) .
\end{aligned}
$$

Thus, the FOC is satisfied if and only if $z_{b}\left(t^{*}\right)=z_{r}\left(t^{*}\right)$. According to equations (2) and (3),

$$
z_{b}\left(t^{*}\right)=z_{r}\left(t^{*}\right) \Longleftrightarrow z_{b}(0)=z_{r}(0) \Longleftrightarrow \frac{p_{b}}{v_{b}}=\frac{p_{r}}{v_{r}} .
$$

To determine the second derivative, we differentiate the first derivative, i.e.,

$$
\begin{aligned}
\frac{\partial M}{\partial v_{b}} & =\left(1-e^{-z_{b}\left(t^{*}\right)}-z_{b}\left(t^{*}\right) e^{-z_{b}\left(t^{*}\right)}\right)-\left(1-e^{-z_{r}\left(t^{*}\right)}-z_{r}\left(t^{*}\right) e^{-z_{r}\left(t^{*}\right)}\right), \\
\frac{\partial^{2} M}{\partial\left(v_{b}\right)^{2}} & =z_{b}\left(t^{*}\right) e^{-z_{b}\left(t^{*}\right)}\left(\frac{d z_{b}}{d v_{b}}-\frac{d z_{b}}{d v_{r}}\right)+z_{r}\left(t^{*}\right) e^{-z_{r}\left(t^{*}\right)}\left(\frac{d z_{r}}{d v_{r}}-\frac{d z_{r}}{d v_{b}}\right)
\end{aligned}
$$


Substituting $\left(\frac{d z_{b}}{d v_{b}}-\frac{d z_{b}}{d v_{r}}\right)$ and $\left(\frac{d z_{r}}{d v_{r}}-\frac{d z_{r}}{d v_{b}}\right)$ implies,

$$
\begin{aligned}
\frac{\partial^{2} M}{\partial\left(v_{b}\right)^{2}} & =\frac{z_{b}\left(t^{*}\right) e^{-z_{b}\left(t^{*}\right)}}{B_{z_{b}}^{\prime} R_{z_{r}}^{\prime}-B_{z_{r}}^{\prime} R_{z_{b}}^{\prime}} p_{r} \frac{a}{a-1} \frac{1-e^{-z_{r}\left(t^{*}\right)}}{z_{r}(0)} \Psi\left(\frac{1}{v_{r}}+\frac{1}{v_{b}}\right) \\
& +\frac{z_{b}\left(t^{*}\right) e^{-z_{b}\left(t^{*}\right)}}{B_{z_{b}}^{\prime} R_{z_{r}}^{\prime}-B_{z_{r}}^{\prime} R_{z_{b}}^{\prime}}\left(\frac{p_{b}}{v_{b}}-\frac{p_{r}}{v_{r}}\right) \frac{a}{a-1} \Psi \frac{1-e^{-z_{r}\left(t^{*}\right)}+z_{r}\left(t^{*}\right) e^{-z_{r}\left(t^{*}\right)}}{z_{r}(0)} \\
& -\frac{z_{b}\left(t^{*}\right) e^{-z_{b}\left(t^{*}\right)}}{B_{z_{b}}^{\prime} R_{z_{r}}^{\prime}-B_{z_{r}}^{\prime} R_{z_{b}}^{\prime}} \frac{1}{v_{b}} \frac{z_{b}\left(t^{*}\right)\left(1-e^{-z_{b}\left(t^{*}\right)}\right)}{z_{b}(0)} \frac{1-e^{-z_{r}\left(t^{*}\right)}+z_{r}\left(t^{*}\right) e^{-z_{r}\left(t^{*}\right)}}{z_{r}(0)} \\
& +\frac{z_{r}\left(t^{*}\right) e^{-z_{r}\left(t^{*}\right)}}{B_{z_{b}}^{\prime} R_{z_{r}}^{\prime}-B_{z_{r}}^{\prime} R_{z_{b}}^{\prime}} p_{b} \frac{a}{a-1} \frac{1-e^{-z_{b}\left(t^{*}\right)}}{z_{b}(0)} \Psi\left(\frac{1}{v_{r}}+\frac{1}{v_{b}}\right) \\
& +\frac{z_{r}\left(t^{*}\right) e^{-z_{r}\left(t^{*}\right)}}{B_{z_{b}}^{\prime} R_{z_{r}}^{\prime}-B_{z_{r}}^{\prime} R_{z_{b}}^{\prime}}\left(\frac{p_{r}}{v_{r}}-\frac{p_{b}}{v_{b}}\right) \frac{a}{a-1} \Psi \frac{1-e^{-z_{b}\left(t^{*}\right)}+z_{b}\left(t^{*}\right) e^{-z_{b}\left(t^{*}\right)}}{z_{b}(0)} \\
& -\frac{z_{r}\left(t^{*}\right) e^{-z_{r}\left(t^{*}\right)}}{B_{z_{b}}^{\prime} R_{z_{r}}^{\prime}-B_{z_{r}}^{\prime} R_{z_{b}}^{\prime}} \frac{1}{v_{r}} \frac{z_{r}\left(t^{*}\right)\left(1-e^{-z_{r}\left(t^{*}\right)}\right)}{z_{r}(0)} \frac{1-e^{-z_{b}\left(t^{*}\right)}+z_{b}\left(t^{*}\right) e^{-z_{b}\left(t^{*}\right)}}{z_{b}(0)} .
\end{aligned}
$$

Rearranging implies

$$
\begin{aligned}
\frac{\partial^{2} M}{\partial\left(v_{b}\right)^{2}} & =-\frac{z_{b}\left(t^{*}\right) e^{-z_{b}\left(t^{*}\right)}}{B_{z_{b}}^{\prime} R_{z_{r}}^{\prime}-B_{z_{r}}^{\prime} R_{z_{b}}^{\prime}} p_{r}\left(\frac{1}{v_{r}}+\frac{1}{v_{b}}\right) \Psi\left(\frac{z_{r}\left(t^{*}\right) e^{-z_{r}\left(t^{*}\right)}}{z_{r}(0)}-\frac{1}{a-1} \frac{1-e^{-z_{r}\left(t^{*}\right)}}{z_{r}(0)}\right) \\
& +\frac{z_{b}\left(t^{*}\right) e^{-z_{b}\left(t^{*}\right)}}{B_{z_{b}}^{\prime} R_{z_{r}}^{\prime}-B_{z_{r}}^{\prime} R_{z_{b}}^{\prime}}\left(\frac{p_{b}}{v_{b}}-\frac{p_{r}}{v_{r}}\right) \frac{1}{a-1} \Psi \frac{1-e^{-z_{r}\left(t^{*}\right)}+z_{r}\left(t^{*}\right) e^{-z_{r}\left(t^{*}\right)}}{z_{r}(0)} \\
& -\frac{z_{r}\left(t^{*}\right) e^{-z_{r}\left(t^{*}\right)}}{B_{z_{b}}^{\prime} R_{z_{r}}^{\prime}-B_{z_{r}}^{\prime} R_{z_{b}}^{\prime}} p_{b}\left(\frac{1}{v_{r}}+\frac{1}{v_{b}}\right) \Psi\left(\frac{z_{b}\left(t^{*}\right) e^{-z_{b}\left(t^{*}\right)}}{z_{b}(0)}-\frac{1}{a-1} \frac{1-e^{-z_{b}\left(t^{*}\right)}}{z_{b}(0)}\right) \\
& +\frac{z_{r}\left(t^{*}\right) e^{-z_{r}\left(t^{*}\right)}}{B_{z_{b}}^{\prime} R_{z_{r}}^{\prime}-B_{z_{r}}^{\prime} R_{z_{b}}^{\prime}}\left(\frac{p_{r}}{v_{r}}-\frac{p_{b}}{v_{b}}\right) \frac{1}{a-1} \Psi \frac{1-e^{-z_{b}\left(t^{*}\right)}+z_{b}\left(t^{*}\right) e^{-z_{b}\left(t^{*}\right)}}{z_{b}(0)}
\end{aligned}
$$

Imposing the FOC, i.e., $\frac{p_{b}}{v_{b}}=\frac{p_{r}}{v_{r}}$, implies that the second derivative is negative, i.e.,

$$
\begin{aligned}
\frac{\partial^{2} M}{\partial\left(v_{b}\right)^{2}} & =-\frac{z_{b}\left(t^{*}\right) e^{-z_{b}\left(t^{*}\right)}}{B_{z_{b}}^{\prime} R_{z_{r}}^{\prime}-B_{z_{r}}^{\prime} R_{z_{b}}^{\prime}}\left(\frac{1}{v_{r}}+\frac{1}{v_{b}}\right) \Psi\left(\frac{z_{r}\left(t^{*}\right) e^{-z_{r}\left(t^{*}\right)}}{z_{r}(0)}-\frac{1}{a-1} \frac{1-e^{-z_{r}\left(t^{*}\right)}}{z_{r}(0)}\right) \\
& =-z_{b}(0) \frac{z_{b}\left(t^{*}\right) e^{-z_{b}\left(t^{*}\right)}}{1-e^{-z_{b}\left(t^{*}\right)}+z_{b}\left(t^{*}\right) e^{-z_{b}\left(t^{*}\right)}}\left(\frac{1}{v_{r}}+\frac{1}{v_{b}}\right) \Psi<0 .
\end{aligned}
$$




\section{B. Comparative statics}

Vacancy creation cost $k$

From equation (14) Proposition 2 in the main text we know that an increase in vacancy creation costs increases the queue length $z\left(t^{*}\right)$ at the end of Phase 1, i.e., $d z\left(t^{*}\right) / d k>0$. Next we show that the number of applications $a^{*}$ decrease with higher vacancy creation costs $k$. We start with applying the Implicit Function Theorem to the optimality condition for the number of applications given in equation (16), i.e.,

$$
\begin{aligned}
G & =-\left(1-e^{-z\left(t^{*}\right)}\right)^{a^{*}} \ln \left(1-e^{-z\left(t^{*}\right)}\right)=h, \\
G_{a}^{\prime} & =-\left(1-e^{-z\left(t^{*}\right)}\right)^{a^{*}}\left(\ln \left(1-e^{-z\left(t^{*}\right)}\right)\right)^{2}, \\
G_{z\left(t^{*}\right)}^{\prime} & =\left(-a^{*} \ln \left(1-e^{-z\left(t^{*}\right)}\right)-1\right) e^{-z\left(t^{*}\right)}\left(1-e^{-z\left(t^{*}\right)}\right)^{\left(a^{*}-1\right)}, \\
\frac{d a^{*}}{d z\left(t^{*}\right)} & =\frac{-\left(a^{*} \ln \left(1-e^{-z\left(t^{*}\right)}\right)+1\right) e^{-z\left(t^{*}\right)}}{\left(1-e^{-z\left(t^{*}\right)}\right)\left(\ln \left(1-e^{-z\left(t^{*}\right)}\right)\right)^{2}}
\end{aligned}
$$

The proof is by contradiction. We want to prove $d a^{*} / d z\left(t^{*}\right)<0$. Suppose now the opposite, i.e.,

$$
\frac{d a^{*}}{d z\left(t^{*}\right)} \geq 0 \Longleftrightarrow-a^{*} \ln \left(1-e^{-z\left(t^{*}\right)}\right) \geq 1
$$

We can define the queue length $z_{a}\left(t^{*}\right)$ implicitly by $1=-a^{*} \ln \left(1-e^{-z_{a}\left(t^{*}\right)}\right)$. Thus, the threshold queue length $\bar{z}_{a}\left(t^{*}\right)$ for the upper bound on the optimal number of applications implied by condition (19) in the main text on the application cost $h$, i.e., $a^{*}=\Phi\left(z_{a}\left(t^{*}\right)\right)$, is given by,

$$
-\Phi\left(\bar{z}_{a}\left(t^{*}\right)\right) \ln \left(1-e^{-\bar{z}_{a}\left(t^{*}\right)}\right)=1 \Longleftrightarrow \bar{z}_{a}\left(t^{*}\right) \approx 1.0874
$$

Since $d a^{*} / d z\left(t^{*}\right) \geq 0$ by assumption we need $z\left(t^{*}\right)<\bar{z}_{a}\left(t^{*}\right) \approx 1.0874$ to ensure $-a^{*} \ln \left(1-e^{-z\left(t^{*}\right)}\right) \geq 1$. But existence requires $z\left(t^{*}\right) \geq \underline{z}\left(t^{*}\right) \approx 1.2628$ as stated in condition (18) in the main text. This leads to the desired contradiction. Hence the number of applications decreases with higher vacancy creation costs, i.e., $d a^{*} / d z\left(t^{*}\right)<0$.

The number of unemployed that participate in the labor market strictly decreases with higher vacancy creation costs $k$ (higher queue length $z\left(t^{*}\right)$ at the end of Phase 1), i.e.,

$$
\frac{d \bar{x}^{*}}{d z\left(t^{*}\right)}=-a^{*} e^{-z\left(t^{*}\right)}\left(1-e^{-z\left(t^{*}\right)}\right)^{\left(a^{*}-1\right)}<0,
$$


where the effect via the number of applications drops out due to the Envelope Theorem. The number of vacancies $v^{*}$ is given by equation (17) in the main text, i.e.,

$$
v^{*}=a^{*} u^{*} \frac{\left(1-e^{-z\left(t^{*}\right)}\right)^{\left(a^{*}-1\right)}}{z\left(t^{*}\right)} .
$$

Differentiating with respect to $z\left(t^{*}\right)$ implies,

$$
\begin{aligned}
\frac{d v^{*}}{d z\left(t^{*}\right)} & =\frac{d a^{*}}{d z\left(t^{*}\right)} \frac{u^{*}\left(1-e^{-z\left(t^{*}\right)}\right)^{\left(a^{*}-1\right)}}{z\left(t^{*}\right)}+\frac{d u^{*}}{d z\left(t^{*}\right)} \frac{a^{*}\left(1-e^{-z\left(t^{*}\right)}\right)^{\left(a^{*}-1\right)}}{z\left(t^{*}\right)} \\
& -a^{*} u^{*} \frac{\left(1-e^{-z\left(t^{*}\right)}\right)-\left(a^{*}-1\right) z\left(t^{*}\right) e^{-z\left(t^{*}\right)}}{\left(1-e^{-z\left(t^{*}\right)}\right) z\left(t^{*}\right)} \frac{\left(1-e^{-z\left(t^{*}\right)}\right)^{\left(a^{*}-1\right)}}{z\left(t^{*}\right)} \\
& +a^{*} u^{*} \frac{\left(1-e^{-z\left(t^{*}\right)}\right)^{\left(a^{*}-1\right)}}{z\left(t^{*}\right)} \ln \left(1-e^{-z\left(t^{*}\right)}\right) \frac{d a^{*}}{d z\left(t^{*}\right)} \\
\frac{d v^{*}}{d z\left(t^{*}\right)} \frac{1}{v^{*}} & =\frac{d a^{*}}{d z\left(t^{*}\right)} \frac{1+a^{*} \ln \left(1-e^{-z\left(t^{*}\right)}\right)}{a^{*}}+\frac{d u^{*}}{d z\left(t^{*}\right)} \frac{1}{u^{*}} \\
& -\frac{\left(1-e^{-z\left(t^{*}\right)}\right)-\left(a^{*}-1\right) z\left(t^{*}\right) e^{-z\left(t^{*}\right)}}{\left(1-e^{-z\left(t^{*}\right)}\right) z\left(t^{*}\right)} \\
& <0,
\end{aligned}
$$

where condition (6) in the main text implies that the second line is negative. In addition $a^{*} \ln \left(1-e^{-z\left(t^{*}\right)}\right)+1>0$, as we have shown in the comparative statics analysis of the number of applications with respect to the vacancy creation costs. Thus, the number of vacancies $v^{*}$ decreases if vacancy creation costs $k$ increase. The number of matches decrease as well with higher vacancy creation costs, i.e.,

$$
\begin{aligned}
M\left(v^{*}, u^{*}, a^{*}\right) & =u^{*}\left(1-\left(1-e^{-z\left(t^{*}\right)}\right)^{a^{*}}\right)+v^{*}\left(1-e^{-z\left(t^{*}\right)}-z\left(t^{*}\right) e^{-z\left(t^{*}\right)}\right) \\
\frac{d M\left(v^{*}, u^{*}, a^{*}\right)}{d z\left(t^{*}\right)} & =\left(1-\left(1-e^{-z\left(t^{*}\right)}\right)^{a^{*}}\right) \frac{d u^{*}}{d z\left(t^{*}\right)}-u^{*} a^{*}\left(1-e^{-z\left(t^{*}\right)}\right)^{\left(a^{*}-1\right)} e^{-z\left(t^{*}\right)} \\
& -u^{*}\left(1-e^{-z\left(t^{*}\right)}\right)^{a^{*}} \ln \left(1-e^{-z\left(t^{*}\right)}\right) \frac{d a^{*}}{d z\left(t^{*}\right)} \\
& +\left(1-e^{-z\left(t^{*}\right)}-z\left(t^{*}\right) e^{-z\left(t^{*}\right)}\right) \frac{d v^{*}}{d z\left(t^{*}\right)}+v^{*} z\left(t^{*}\right) e^{-z\left(t^{*}\right)} \\
& =\left(1-\left(1-e^{-z\left(t^{*}\right)}\right)^{a}\right) \frac{d u^{*}}{d z\left(t^{*}\right)}-u^{*}\left(1-e^{-z\left(t^{*}\right)}\right)^{a} \ln \left(1-e^{-z\left(t^{*}\right)}\right) \frac{d a^{*}}{d z\left(t^{*}\right)} \\
& +\left(1-e^{-z\left(t^{*}\right)}-z\left(t^{*}\right) e^{-z\left(t^{*}\right)}\right) \frac{d v^{*}}{d z\left(t^{*}\right)} \\
& <0
\end{aligned}
$$


The matching probability of firms increases, i.e.,

$$
\begin{aligned}
\frac{M\left(v^{*}, u^{*}, a^{*}\right)}{v^{*}} & =\frac{u^{*}}{v^{*}}\left(1-\left(1-e^{-z\left(t^{*}\right)}\right)^{a *}\right)+\left(1-e^{-z\left(t^{*}\right)}-z\left(t^{*}\right) e^{-z\left(t^{*}\right)}\right) \\
\frac{d\left(M\left(v^{*}, u^{*}, a^{*}\right) / v^{*}\right)}{d z\left(t^{*}\right)} & =\left(1-\left(1-e^{-z\left(t^{*}\right)}\right)^{a^{*}}\right)\left(\frac{d u^{*}}{d z\left(t^{*}\right)} \frac{1}{v^{*}}-\frac{u^{*}}{\left(v^{*}\right)} \frac{d v^{*}}{d z\left(t^{*}\right)}\right) \\
& -\frac{u^{*} a^{*}}{v^{*}}\left(1-e^{-z\left(t^{*}\right)}\right){ }^{\left(a^{*}-1\right)} e^{-z\left(t^{*}\right)} \\
& -\frac{u^{*}}{v^{*}}\left(1-e^{-z\left(t^{*}\right)}\right)^{a^{*}} \ln \left(1-e^{-z\left(t^{*}\right)}\right) \frac{d a^{*}}{d z\left(t^{*}\right)}+z\left(t^{*}\right) e^{-z\left(t^{*}\right)} \\
& =\left(1-\left(1-e^{-z\left(t^{*}\right)}\right)^{a^{*}}\right)\left(\frac{d u^{*}}{d z\left(t^{*}\right)} \frac{1}{v^{*}}-\frac{u^{*}}{\left(v^{*}\right)} \frac{d v^{*}}{d z\left(t^{*}\right)}\right) \\
& -\frac{u^{*}}{v^{*}}\left(1-e^{-z\left(t^{*}\right)}\right)^{a^{*}} \ln \left(1-e^{-z\left(t^{*}\right)}\right) \frac{d a^{*}}{d z\left(t^{*}\right)} \\
& >0
\end{aligned}
$$

where we used equation (8) to get,

$$
\begin{aligned}
& \frac{d u^{*}}{d z\left(t^{*}\right)} \frac{1}{v^{*}}-\frac{u^{*}}{\left(v^{*}\right)^{2}} \frac{d v^{*}}{d z\left(t^{*}\right)} \\
& =-\frac{d a^{*}}{d z\left(t^{*}\right)} \frac{1+a^{*} \ln \left(1-e^{-z\left(t^{*}\right)}\right)}{a^{*}} \frac{u^{*}}{v^{*}}+\frac{u^{*}}{v^{*}} \frac{\left(1-e^{-z\left(t^{*}\right)}\right)-\left(a^{*}-1\right) z\left(t^{*}\right) e^{-z\left(t^{*}\right)}}{\left(1-e^{-z\left(t^{*}\right)}\right) z\left(t^{*}\right)}>0 .
\end{aligned}
$$

The matching probability of workers decreases, i.e.,

$$
\begin{aligned}
\frac{M\left(v^{*}, u^{*}, a^{*}\right)}{u} & =\left(1-\left(1-e^{-z\left(t^{*}\right)}\right)^{a^{*}}\right)+\frac{v^{*}}{u^{*}}\left(1-e^{-z\left(t^{*}\right)}-z\left(t^{*}\right) e^{-z\left(t^{*}\right)}\right) \\
\frac{d\left(M\left(v^{*}, u^{*}, a^{*}\right) / u^{*}\right)}{d z\left(t^{*}\right)} & =-a^{*}\left(1-e^{-z\left(t^{*}\right)}\right)^{\left(a^{*}-1\right)} e^{-z\left(t^{*}\right)}-\left(1-e^{-z\left(t^{*}\right)}\right)^{a^{*}} \ln \left(1-e^{-z\left(t^{*}\right)}\right) \frac{d a^{*}}{d z\left(t^{*}\right)} \\
& +\left(1-e^{-z\left(t^{*}\right)}-z\left(t^{*}\right) e^{-z\left(t^{*}\right)}\right)\left(\frac{d v^{*}}{d z\left(t^{*}\right)} \frac{1}{u^{*}}-\frac{d u^{*}}{d z\left(t^{*}\right)} \frac{v^{*}}{\left(u^{*}\right)^{2}}\right)+\frac{v^{*}}{u^{*}} z\left(t^{*}\right) e^{-z\left(t^{*}\right)} \\
& =-\left(1-e^{-z\left(t^{*}\right)}\right)^{a^{*}} \ln \left(1-e^{-z\left(t^{*}\right)}\right) \frac{d a^{*}}{d z\left(t^{*}\right)} \\
& -\left(1-e^{-z\left(t^{*}\right)}-z\left(t^{*}\right) e^{-z\left(t^{*}\right)}\right)\left(\frac{d u^{*}}{d z\left(t^{*}\right)} \frac{v^{*}}{\left(u^{*}\right)^{2}}-\frac{d v^{*}}{d z\left(t^{*}\right)} \frac{1}{u^{*}}\right) \\
& <0
\end{aligned}
$$


where we used equation (8) to get,

$$
\begin{aligned}
& \frac{d u^{*}}{d z\left(t^{*}\right)} \frac{v^{*}}{\left(u^{*}\right)^{2}}-\frac{d v^{*}}{d z\left(t^{*}\right)} \frac{1}{u^{*}} \\
& =-\frac{d a^{*}}{d z\left(t^{*}\right)} \frac{1+a^{*} \ln \left(1-e^{-z\left(t^{*}\right)}\right)}{a^{*}} \frac{v^{*}}{u^{*}}+\frac{v^{*}}{u^{*}} \frac{\left(1-e^{-z\left(t^{*}\right)}\right)-\left(a^{*}-1\right) z\left(t^{*}\right) e^{-z\left(t^{*}\right)}}{\left(1-e^{-z\left(t^{*}\right)}\right) z\left(t^{*}\right)}>0 .
\end{aligned}
$$

The effect on the average wage is ambiguous, as one can see by differentiating $E[w]$ with respect to $z\left(t^{*}\right)$, i.e.,

$$
\begin{aligned}
E[w] & =\frac{u^{*}\left(1-\left(1-e^{-z\left(t^{*}\right)}\right)^{a^{*}}\right)}{u^{*}\left(1-\left(1-e^{-z\left(t^{*}\right)}\right)^{a^{*}}\right)+v^{*}\left(1-e^{-z\left(t^{*}\right)}-z\left(t^{*}\right) e^{-z\left(t^{*}\right)}\right)} \\
\frac{d E[w]}{d z\left(t^{*}\right)} & =\frac{u^{*}\left(1-\left(1-e^{-z\left(t^{*}\right)}\right)^{a^{*}}\right) v^{*}\left(1-e^{-z\left(t^{*}\right)}-z\left(t^{*}\right) e^{-z\left(t^{*}\right)}\right)}{M\left(v^{*}, u^{*}, a^{*}\right)^{2}}\left(\frac{d u^{*}}{d z\left(t^{*}\right)} \frac{1}{u^{*}}-\frac{d v^{*}}{d z\left(t^{*}\right)} \frac{1}{v^{*}}\right) \\
& -\frac{u^{*}\left(1-e^{-z\left(t^{*}\right)}\right)^{a^{*}} \ln \left(1-e^{-z\left(t^{*}\right)}\right) v^{*}\left(1-e^{-z\left(t^{*}\right)}-z\left(t^{*}\right) e^{-z\left(t^{*}\right)}\right)}{M\left(v^{*}, u^{*}, a^{*}\right)^{2}} \frac{d a^{*}}{d z\left(t^{*}\right)} \\
& -\frac{u^{*} a^{*}\left(1-e^{-z\left(t^{*}\right)}\right)^{a^{*}-1} e^{-z\left(t^{*}\right)}}{M\left(v^{*}, u^{*}, a^{*}\right)} \\
& =E[w](1-E[w])\left(\frac{d u^{*}}{d z\left(t^{*}\right)} \frac{1}{u^{*}}-\frac{d v^{*}}{d z\left(t^{*}\right)} \frac{1}{v^{*}}-\frac{\left(1-e^{-z\left(t^{*}\right)}\right)^{a^{*}} \ln \left(1-e^{-z\left(t^{*}\right)}\right)}{\left(1-\left(1-e^{-z\left(t^{*}\right)}\right)^{a^{*}}\right)} \frac{d a^{*}}{d z\left(t^{*}\right)}\right) \\
& -\frac{u^{*} a^{*}\left(1-e^{-z\left(t^{*}\right)}\right)^{a^{*}-1} e^{-z\left(t^{*}\right)}}{M\left(v^{*}, u^{*}, a^{*}\right)}
\end{aligned}
$$

since

$\frac{d u^{*}}{d z\left(t^{*}\right)} \frac{1}{u^{*}}-\frac{d v^{*}}{d z\left(t^{*}\right)} \frac{1}{v^{*}}=-\frac{d a^{*}}{d z\left(t^{*}\right)} \frac{1+a^{*} \ln \left(1-e^{-z\left(t^{*}\right)}\right)}{a^{*}}+\frac{\left(1-e^{-z\left(t^{*}\right)}\right)-\left(a^{*}-1\right) z\left(t^{*}\right) e^{-z\left(t^{*}\right)}}{\left(1-e^{-z\left(t^{*}\right)}\right) z\left(t^{*}\right)}>0$ 
Application cost $h$

From equation (14) Proposition 2 we know that an increase in application costs has no effect on the queue length $z\left(t^{*}\right)$ at the end of Phase 1, i.e., $d z\left(t^{*}\right) / d h=0$. We first show that the number of applications $a^{*}$ decrease with higher application cost $h$ as one can easily show applying the Implicit Function Theorem to the optimality condition for the number of applications given in equation (16), i.e.,

$$
\begin{aligned}
G & =-\left(1-e^{-z\left(t^{*}\right)}\right)^{a^{*}} \ln \left(1-e^{-z\left(t^{*}\right)}\right)-h, \\
G_{a}^{\prime} & =-\left(1-e^{-z\left(t^{*}\right)}\right)^{a^{*}}\left(\ln \left(1-e^{-z\left(t^{*}\right)}\right)\right)^{2}, \\
G_{h}^{\prime} & =-1, \\
\frac{d a^{*}}{d h} & =\frac{-1}{\left(1-e^{-z\left(t^{*}\right)}\right)\left(\ln \left(1-e^{-z\left(t^{*}\right)}\right)\right)^{2}}<0,
\end{aligned}
$$

The number of unemployed that participate in the labor market strictly decreases with higher application cost $h$, i.e.,

$$
\bar{x}^{*}=1-\left(1-e^{-z\left(t^{*}\right)}\right)^{a^{*}}-h a^{*} \Longrightarrow \frac{\partial \bar{x}^{*}}{\partial h}=-a^{*}<0,
$$

where the effect via the number of applications drops out due to the Envelope Theorem. The number of vacancies $v^{*}$ is given by equation (17), i.e.,

$$
v^{*}=a^{*} u^{*} \frac{\left(1-e^{-z\left(t^{*}\right)}\right)^{\left(a^{*}-1\right)}}{z\left(t^{*}\right)} .
$$

Differentiating with respect to $h$ implies that the number of vacancies $v^{*}$ decreases with $h$, i.e.,

$$
\begin{aligned}
\frac{d v^{*}}{d h} & =\frac{d a^{*}}{d h} \frac{u^{*}\left(1-e^{-z\left(t^{*}\right)}\right)^{\left(a^{*}-1\right)}}{z\left(t^{*}\right)}+\frac{d u^{*}}{d h} \frac{a^{*}\left(1-e^{-z\left(t^{*}\right)}\right)^{\left(a^{*}-1\right)}}{z\left(t^{*}\right)} \\
& +a^{*} u^{*} \frac{\left(1-e^{-z\left(t^{*}\right)}\right)^{\left(a^{*}-1\right)}}{z\left(t^{*}\right)} \ln \left(1-e^{-z\left(t^{*}\right)}\right) \\
& <0 .
\end{aligned}
$$

The number of matches decrease as well with higher application costs $h$, i.e.,

$$
\begin{aligned}
\frac{d M\left(v^{*}, u^{*}, a^{*}\right)}{d h} & =\left(1-\left(1-e^{-z\left(t^{*}\right)}\right)^{a^{*}}\right) \frac{d u^{*}}{d h}-u^{*}\left(1-e^{-z\left(t^{*}\right)}\right)^{a^{*}} \ln \left(1-e^{-z\left(t^{*}\right)}\right) \frac{d a^{*}}{d h} \\
& +\left(1-e^{-z\left(t^{*}\right)}-z\left(t^{*}\right) e^{-z\left(t^{*}\right)}\right) \frac{d v^{*}}{d h} \\
& <0 .
\end{aligned}
$$


The matching probability of firms increases, i.e.,

$$
\begin{aligned}
\frac{d\left(M\left(v^{*}, u^{*}, a^{*}\right) / v^{*}\right)}{d h} & =\left(1-\left(1-e^{-z\left(t^{*}\right)}\right)^{a^{*}}\right)\left(\frac{d u^{*}}{d h} \frac{1}{v^{*}}-\frac{u^{*}}{\left(v^{*}\right)^{2}} \frac{d v^{*}}{d h}\right) \\
& -\frac{u^{*}}{v^{*}}\left(1-e^{-z\left(t^{*}\right)}\right)^{a^{*}} \ln \left(1-e^{-z\left(t^{*}\right)}\right) \frac{d a^{*}}{d h} \\
& =\left(1-\left(1-e^{-z\left(t^{*}\right)}\right)^{a^{*}}\right) \frac{u^{*}}{v^{*}}\left(-\frac{1}{a^{*}} \frac{d a^{*}}{d h}-\ln \left(1-e^{-z\left(t^{*}\right)}\right)\right) \\
& -\frac{u^{*}}{v^{*}}\left(1-e^{-z\left(t^{*}\right)}\right)^{a^{*}} \ln \left(1-e^{-z\left(t^{*}\right)}\right) \frac{d a^{*}}{d h} \\
& \left.=-\bar{x}^{*} \frac{u^{*}}{v^{*}} \frac{1}{a^{*}} \frac{d a^{*}}{d h}-\left(1-\left(1-e^{-z\left(t^{*}\right)}\right)\right)^{a^{*}}\right) \frac{u^{*}}{v^{*}} \ln \left(1-e^{-z\left(t^{*}\right)}\right) \\
& >0,
\end{aligned}
$$

and the matching probability of workers decreases, i.e.,

$$
\begin{aligned}
\frac{d\left(M\left(v^{*}, u^{*}, a^{*}\right) / u^{*}\right)}{d h} & =-\left(1-e^{-z\left(t^{*}\right)}\right)^{a^{*}} \ln \left(1-e^{-z\left(t^{*}\right)}\right) \frac{d a^{*}}{d h} \\
& +\frac{v^{*}}{u^{*}}\left(1-e^{-z\left(t^{*}\right)}-z\left(t^{*}\right) e^{-z\left(t^{*}\right)}\right)\left(\frac{d v^{*}}{d h} \frac{1}{v^{*}}-\frac{d u^{*}}{d h} \frac{1}{u^{*}}\right) \\
& <0,
\end{aligned}
$$

since equation (9) implies

$$
\frac{1}{v^{*}} \frac{d v^{*}}{d h}-\frac{1}{u^{*}} \frac{d u^{*}}{d h}=\frac{1}{a^{*}} \frac{d a^{*}}{d h}+\ln \left(1-e^{-z\left(t^{*}\right)}\right)<0 .
$$

The average wage increases with higher application cost $h$. This can be seen by noting that the average wage can be written as,

$$
E[w]=\frac{\left(M\left(v^{*}, u^{*}, a^{*}\right) / v^{*}\right)-\left(1-e^{-z\left(t^{*}\right)}-z\left(t^{*}\right) e^{-z\left(t^{*}\right)}\right)}{\left(M\left(v^{*}, u^{*}, a^{*}\right) / v^{*}\right)},
$$

where differentiating (using the fact that $z\left(t^{*}\right)$ does not change) implies,

$$
\frac{d E[w]}{d h}\left(\frac{M\left(v^{*}, u^{*}, a^{*}\right)}{v^{*}}\right)^{2}=\frac{d\left(M\left(v^{*}, u^{*}, a^{*}\right) / v^{*}\right)}{d h}\left(1-e^{-z\left(t^{*}\right)}-z\left(t^{*}\right) e^{-z\left(t^{*}\right)}\right)>0 .
$$


Match value $y$

Introducing a match value $y$ into the model implies,

$$
\begin{aligned}
y\left(1-e^{-z\left(t^{*}\right)}-z\left(t^{*}\right) e^{-z\left(t^{*}\right)}\right) & =k, \\
y\left(1-\left(1-e^{-z\left(t^{*}\right)}\right)^{a^{*}}\right) & =h a^{*}+\bar{x}^{*} \\
-y\left(1-e^{-z\left(t^{*}\right)}\right)^{a^{*}} \ln \left(1-e^{-z\left(t^{*}\right)}\right) & =h,
\end{aligned}
$$

where

$$
\frac{v^{*}}{a^{*} u^{*}}=\frac{1}{z\left(t^{*}\right)}\left(1-e^{-z\left(t^{*}\right)}\right)^{a^{*}-1} .
$$

From equation (10) we know that an increase in productivity decreases the queue length $z\left(t^{*}\right)$ at the end of Phase 1, i.e.,

$$
\frac{d z\left(t^{*}\right)}{d y}=-\frac{\left(1-e^{-z\left(t^{*}\right)}-z\left(t^{*}\right) e^{-z\left(t^{*}\right)}\right)}{y z\left(t^{*}\right) e^{-z\left(t^{*}\right)}}<0
$$

Next we show that the number of applications $a^{*}$ increase with a higher match value $y$. We start with applying the Implicit Function Theorem to the optimality condition for the number of applications given in equation (12), i.e.,

$$
\begin{aligned}
G & =-y\left(1-e^{-z\left(t^{*}\right)}\right)^{a^{*}} \ln \left(1-e^{-z\left(t^{*}\right)}\right)=h \\
G_{a}^{\prime} & =-y\left(1-e^{-z\left(t^{*}\right)}\right)^{a^{*}}\left(\ln \left(1-e^{-z\left(t^{*}\right)}\right)\right)^{2} \\
G_{y}^{\prime} & =-\left(1-e^{-z\left(t^{*}\right)}\right)^{a^{*}} \ln \left(1-e^{-z\left(t^{*}\right)}\right) \\
& +y\left(-a^{*} \ln \left(1-e^{-z\left(t^{*}\right)}\right)-1\right) e^{-z\left(t^{*}\right)}\left(1-e^{-z\left(t^{*}\right)}\right)^{\left(a^{*}-1\right)} \frac{d z\left(t^{*}\right)}{d y} \\
\frac{d a^{*}}{d y} & =\frac{-\ln \left(1-e^{-z\left(t^{*}\right)}\right)+\left(a^{*} \ln \left(1-e^{-z\left(t^{*}\right)}\right)+1\right) \frac{\left(1-e^{-z\left(t^{*}\right)}-z\left(t^{*}\right) e^{-z\left(t^{*}\right)}\right)}{z\left(t^{*}\right)\left(1-e^{-z\left(t^{*}\right)}\right)}}{y\left(\ln \left(1-e^{-z\left(t^{*}\right)}\right)\right)^{2}}
\end{aligned}
$$

Using the condition (19) on the application cost $h$ as restrictions on $a^{*}$ we get that the number of applications increases with productivity at the lower and 
upper bound of $a^{*}$, i.e.,

$$
\begin{aligned}
\left.\frac{d a^{*}}{d y}\right|_{a^{*}=\Phi\left(z\left(t^{*}\right)\right)} & =\frac{\left(1-e^{-z\left(t^{*}\right)}-z\left(t^{*}\right) e^{-z\left(t^{*}\right)}\right)}{z\left(t^{*}\right)\left(1-e^{-z\left(t^{*}\right)}\right)}>0, \\
\left.\frac{d a^{*}}{d y}\right|_{a^{*}=1} & =\left(\frac{1-e^{-z\left(t^{*}\right)}}{z\left(t^{*}\right)}-1\right) \frac{\ln \left(1-e^{-z\left(t^{*}\right)}\right)}{1-e^{-z\left(t^{*}\right)}}+\frac{\left(1-e^{-z\left(t^{*}\right)}-z\left(t^{*}\right) e^{-z\left(t^{*}\right)}\right)}{z\left(t^{*}\right)\left(1-e^{-z\left(t^{*}\right)}\right)}>0 .
\end{aligned}
$$

Furthermore, the derivative $d a^{*} / d y$ is strictly decreasing in $a^{*}$, i.e.,

$$
\frac{d}{d a^{*}}\left[\frac{d a^{*}}{d y}\right]=\ln \left(1-e^{-z\left(t^{*}\right)}\right) \frac{\left(1-e^{-z\left(t^{*}\right)}-z\left(t^{*}\right) e^{-z\left(t^{*}\right)}\right)}{z\left(t^{*}\right)\left(1-e^{-z\left(t^{*}\right)}\right)}<0
$$

which implies $d a^{*} / d y>0$ for any $1 \geq a^{*} \geq \Phi\left(z\left(t^{*}\right)\right)$.

The number of unemployed that participate in the labor market strictly increases with a higher match value $y$, i.e.,

$$
\frac{d \bar{x}^{*}}{d y}=1-\left(1-e^{-z\left(t^{*}\right)}\right)^{a^{*}}-y a^{*} e^{-z\left(t^{*}\right)}\left(1-e^{-z\left(t^{*}\right)}\right)^{\left(a^{*}-1\right)} \frac{d z\left(t^{*}\right)}{d y}>0,
$$

where the effect via the number of applications drops out due to the Envelope Theorem.

The number of vacancies $v^{*}$ is given by equation (17), i.e.,

$$
v^{*}=a^{*} u^{*} \frac{\left(1-e^{-z\left(t^{*}\right)}\right)^{\left(a^{*}-1\right)}}{z\left(t^{*}\right)} .
$$

Differentiating with respect to the match value $y$ implies,

$$
\begin{aligned}
\frac{d v^{*}}{d y} \frac{1}{v^{*}} & =\frac{d a^{*}}{d y} \frac{1}{a^{*}} \frac{a^{*} u^{*}\left(1-e^{-z\left(t^{*}\right)}\right)^{\left(a^{*}-1\right)}}{v^{*} z\left(t^{*}\right)}+\frac{d u^{*}}{d y} \frac{1}{u^{*}} \frac{a^{*} u^{*}\left(1-e^{-z\left(t^{*}\right)}\right)^{\left(a^{*}-1\right)}}{v^{*} z\left(t^{*}\right)} \\
& -\frac{a^{*} u^{*}}{v^{*}} \frac{\left(1-e^{-z\left(t^{*}\right)}\right)-\left(a^{*}-1\right) z\left(t^{*}\right) e^{-z\left(t^{*}\right)}}{\left(1-e^{-z\left(t^{*}\right)}\right) z\left(t^{*}\right)} \frac{\left(1-e^{-z\left(t^{*}\right)}\right)\left(a^{*}-1\right)}{z\left(t^{*}\right)} \frac{d z\left(t^{*}\right)}{d y} \\
& +\frac{a^{*} u^{*}}{v^{*}} \frac{\left(1-e^{-z\left(t^{*}\right)}\right)\left(a^{*}-1\right)}{z\left(t^{*}\right)} \ln \left(1-e^{-z\left(t^{*}\right)}\right) \frac{d a^{*}}{d y} \\
& =\frac{d a^{*}}{d y} \frac{a^{*} \ln \left(1-e^{-z\left(t^{*}\right)}\right)+1}{a^{*}}+\frac{d u^{*}}{d y} \frac{1}{u^{*}} \\
& -\frac{\left(1-e^{-z\left(t^{*}\right)}\right)-\left(a^{*}-1\right) z\left(t^{*}\right) e^{-z\left(t^{*}\right)}}{\left(1-e^{-z\left(t^{*}\right)}\right) z\left(t^{*}\right)} \frac{d z\left(t^{*}\right)}{d y}
\end{aligned}
$$


where condition (6) in the main text implies that the second line is positive. In addition $a^{*} \ln \left(1-e^{-z\left(t^{*}\right)}\right)+1>0$, as we have shown in the comparative statics analysis with respect to the vacancy creation costs. Thus, the number of vacancies $v^{*}$ increases with the value of a match $y$.

The number of matches, the matching probability of workers and the matching probability of firms does not directly depend on the value of the match $y$. They change only with the queue length at the end of Phase $1 z\left(t^{*}\right)$. The derivatives with respect to $z\left(t^{*}\right)$ are the same as for a change in vacancy creation costs. We thus get that the number of matches and the matching probability of workers increase and the matching probability of firms decreases with match quality.

The effect on the average wage is ambiguous, as one can see by differentiating $E[w]$ with respect to the value of the match $y$, i.e.,

$$
\begin{aligned}
E[w] & =y \frac{u^{*}\left(1-\left(1-e^{-z\left(t^{*}\right)}\right)^{a^{*}}\right)}{u^{*}\left(1-\left(1-e^{-z\left(t^{*}\right)}\right)^{a^{*}}\right)+v^{*}\left(1-e^{-z\left(t^{*}\right)}-z\left(t^{*}\right) e^{-z\left(t^{*}\right)}\right)} \\
\frac{d E[w]}{d y} & =\frac{u^{*}\left(1-\left(1-e^{-z\left(t^{*}\right)}\right)^{a^{*}}\right)}{M\left(v^{*}, u^{*}, a^{*}\right)} \\
& +y \frac{u^{*}\left(1-\left(1-e^{-z\left(t^{*}\right)}\right)^{a^{*}}\right) v^{*}\left(1-e^{-z\left(t^{*}\right)}-z\left(t^{*}\right) e^{-z\left(t^{*}\right)}\right)}{M\left(v^{*}, u^{*}, a^{*}\right)^{2}}\left(\frac{d u^{*}}{d y} \frac{1}{u^{*}}-\frac{d v^{*}}{d y} \frac{1}{v^{*}}\right) \\
& +y \frac{-u^{*}\left(1-e^{-z\left(t^{*}\right)}\right)^{a^{*}} \ln \left(1-e^{-z\left(t^{*}\right)}\right) v^{*}\left(1-e^{-z\left(t^{*}\right)}-z\left(t^{*}\right) e^{-z\left(t^{*}\right)}\right)}{M\left(v^{*}, u^{*}, a^{*}\right)^{2}} \frac{d a^{*}}{d y} \\
& +y \frac{-u^{*} a^{*}\left(1-e^{-z\left(t^{*}\right)}\right)^{a^{*}-1} e^{-z\left(t^{*}\right)} \frac{d z\left(t^{*}\right)}{d y}}{M\left(v^{*}, u^{*}, a^{*}\right)} \\
& =1+\frac{1}{y} E[w](1-E[w])\left(\frac{d u^{*}}{d y} \frac{1}{u^{*}}-\frac{d v^{*}}{d y} \frac{1}{v^{*}}\right) \\
& -\frac{1}{y} E[w](1-E[w]) M\left(v^{*}, u^{*}, a^{*}\right) \frac{\left(1-e^{-z\left(t^{*}\right)}\right) a^{*} \ln \left(1-e^{-z\left(t^{*}\right)}\right)}{\left(1-\left(1-e^{-z\left(t^{*}\right)}\right)^{a^{*}}\right)} \frac{d a^{*}}{d y} \\
& \left.=1-\frac{1}{y} E[w](1-E[w]) \frac{\ln \left(1-e^{-z\left(t^{*}\right)}\right)^{a^{*}}+\left(1-\left(1-e^{-z\left(t^{*}\right)}\right)\right.}{a^{*}}\right) \\
& +\frac{1}{y} E[w](1-E[w]) \frac{\left(1-e^{-z\left(t^{*}\right)}\right)-\left(a^{*}-1\right) z\left(t^{*}\right) e^{-z\left(t^{*}\right)}}{d y} \\
& \frac{\left.d 1-e^{-z\left(t^{*}\right)}\right) z\left(t^{*}\right)}{d y}
\end{aligned}
$$

Note that $\ln \left(1-e^{-z\left(t^{*}\right)}\right)^{a^{*}}+\left(1-\left(1-e^{-z\left(t^{*}\right)}\right)^{a^{*}}\right)<0$, since $\ln x+1-x$ is negative for any $x$. The overall effect is therefore ambiguous. 